\title{
Comparison of Vitex agnus-castus Extracts with Placebo in Reducing Menopausal Symptoms: A Randomized Double-Blind Study
}

\author{
Rozita Naseri', Vahid Farnia ${ }^{2}$, Katayoun Yazdchi ${ }^{3 *}$, Mostafa Alikhani $^{2, *}$, Behrad Basanj $^{2}$, Safora Salemi ${ }^{2}$ \\ ${ }^{1}$ Department of Internal Medicine, School of Medicine, Kermanshah University of Medical Sciences, Kermanshah, Iran \\ ${ }^{2}$ Department of Psychiatry, Substance Abuse Prevention Research Center, Health institute, Kermanshah University of Medical Sciences, Kermanshah, \\ Iran \\ ${ }^{3}$ Department of Gynecology and Obstetrics, Kermanshah University Of Medical Sciences, Kermanshah, Iran
}

Background: Menopausal symptoms have remarkable negative effects on women's quality of life, justifying the need to assess various therapeutic options. This research aimed to determine the effectiveness of Vitex agnus-castus extracts in alleviating menopausal symptoms in comparison with that of placebo.

Methods: This study was a randomized controlled double-blind clinical trial with a study group of 52 women referred to a clinic in Kermanshah in 2017. The participants were randomly divided into two groups: Vitex group (26 subjects) and placebo group (26 subjects). Menopausal symptoms were assessed using the Greene Scale before and 8 weeks after the intervention.

Results: After the intervention, the mean scores for total menopausal disorder, anxiety, and vasomotor dysfunction were significantly lower in the Vitex group than in the placebo group $(\mathrm{P}<0.05)$. The mean scores of the variables of somatic complications, depression, and sexual dysfunction did not show significant differences between the Vitex and placebo groups $(\mathrm{P}>0.05)$.

Conclusion: Administration of Vitex agnus-castus extracts as a phytoestrogenic medicine can alleviate menopausal symptoms in women.

Keywords: Vitex agnus-castus Extract; Placebo Effect; Menopause; Women

Received: May 18, 2018, Revised: June 3, 2018, Accepted: June 5, 2018

${ }^{*}$ Corresponding Author: Katayoun Yazdchi https://orcid.org/0000-0002-1742-7313

Tel: +98-9125188875, Fax: +98-83-38260700, E-mail: Kyazdchi@kums.ac.ir

${ }^{*}$ Corresponding Author: Mostafa Alikhani https://orcid.org/0000-0001-6848-3027

Tel: +98-9301192045, Fax: +98-83-38264513,E-mail: Mostafaalikhani18@gmail.com

*These two corresponding authors contributed equally to this study. 


\section{INTRODUCTION}

Menopause is a natural growth transition stage in every woman. ${ }^{1)}$ During menopause, the activities of estrogen and progesterone secreted by the ovaries dramatically decrease. ${ }^{2,3)}$ Women experience physical, mental, social, and psychological changes during menopause, which results in cardiovascular diseases, osteoporosis, bone fracture, and even Alzheimer disease in the long term. ${ }^{4-6)}$ Menopause with complications such as severe hot flash, sudden sweats, fatigue, and dizziness, as well as physiological complications, leads to osteoporosis, ovarian atrophy, decrease in sexual hormone levels, increase in weight, and depression. Thus, acquiring good knowledge about effective treatments to reduce mental and psychological problems of menopausal women is of high value and importance. ${ }^{7-10)}$

Use of various alternative or complementary treatments to reduce the undesirable symptoms of menopause is widely accepted nowadays. Among such treatments, phytoestrogens are used as hormonal therapies in menopausal women; these can have less side effects because of their herbal origin. ${ }^{11)}$ Phytoestrogens are found in soybean, beans, cereals, and vegetables and have an estradiol-like function. ${ }^{12)}$

Administration of herbal medicines has been popular since a long time for the cure of irregular menstruation, menopausal complications, and menstrual pain. In Iranian traditional medicine, several herbal medicines have been suggested to be effective in alleviating menopausal symptoms. Vitex agnus-castus extracts (panj-angosht in Persian) is a native plant of the Mediterranean countries has a long history in traditional medicine. Botanical studies, cultivation, and pharmacognosy of Vitex started in Iran in 1991, and medicinal products were introduced in 1996. ${ }^{13)}$ Rates of complication, including mild upset stomach, rash, and itching, are low; complications are seen in approximately $2 \%$ of users. ${ }^{14-17)}$ Vitex has numerous uses in traditional medicine, and primary studies have demonstrated its effectiveness in decreasing menopausal complications. ${ }^{18-20)}$ The fruit of this plant is administered as treatment for menopausal complaints. Currently, in European and American countries, the use of the Vitex fruit for its therapeutic effects on women with reproductive problems, premenstrual syndrome, infertility, and hyperprolactinemia is common. Despite the lack of clinical trials designed to study the effects of this plant on menopause-related symptoms, pharmacological evidence denoting its dopaminergic activity, closeness to opioid receptors, and ability to increase melatonin secretion support the effectiveness of Vitex for reducing menopausal symptoms. ${ }^{16)}$

Nearly one-third of a woman's lifetime is in the postmenopausal years. Menopause causes various complications; hence, searching for a treatment with less side effects and reasonable effectiveness for menopausal problems seems important and necessary. Herbal medicines have less side effects than chemical medications.

Clinical trials conducted on the efficacy of Vitex in improving menopausal symptoms only examined the general score for menopause based on the Greene Questionnaire. In addition to the total score, relevant symptoms, including anxiety, depression, somatic complications, and vasomotor and sexual dysfunctions, were investigated.

\section{METHODS}

This study was a randomized double-blind clinical trial with a pretestposttest design registered in the Iranian clinical trial website coded as IRCT 2016120528967N3.

\section{Subjects}

The population included all menopausal women referred to the Helae-Ahmar clinic in Kermanshah in 2017 for the treatment of menopausal symptoms. Considering the type of study and the mean and standard deviation of the frequency of hot flashes (extracted from the study performed by Abbaspoor et al. ${ }^{20)}$ on women with menopausal symptoms) within 24 hours and 2 weeks after the beginning of the study, the minimum required sample sizes in the Vitex group (4 \pm 2.52$)$ and control group $(6 \pm 2.34)$ were both 23 . In the calculation, the formula mention below and error level of $5 \%$ also used. Considering that $10 \%$ (three subjects) of subjects would be excluded, the final sample size was determined to be 26 people in each group. The subjects were selected from among the study population on the basis of the inclusion and exclusion criteria of the study.

$$
\begin{aligned}
\mathrm{n} & =\frac{\left(\mathrm{z}_{1-\frac{\alpha}{2}}+\mathrm{z}_{1-\beta}\right)^{2}\left(\sigma_{1}^{2}+\sigma_{2}^{2}\right)}{\left(\mu_{2}-\mu_{1}\right)^{2}}=\left(\frac{1.96+0.85}{6-4}\right)^{2}\left(2.34^{2}+2.52^{2}\right) \\
& =23+3(10 \%)=26
\end{aligned}
$$

The subjects were randomly allocated to the Vitex (treatment group, $\mathrm{n}=26$ ) and placebo groups (control group, $\mathrm{n}=26$ ). The primary outcome was menopausal symptoms (anxiety, depression, somatic complications, and vasomotor and sexual dysfunctions) evaluated using the Greene Questionnaire.

The inclusion criteria were age of 45-65 years, at least 1- to 3-year interval after the last menstruation, at least two hot flashes a day, and a Papanicolaou test result not higher than class II. The exclusion criteria were cigarette smoking; use of hormones or antibiotics in the recent 3 months; systemic diseases such as renal disease, hypertension, high blood sugar, hyperlipidemia, depression, anxiety (depression and anxiety diagnoses based on the 'diagnostic and statistical manual of mental disorders,' fifth edition, criteria made by the psychiatrist); problematic breast examination result; use of antithyroid medications, traditional medicines, gabapentin, selective serotonin reuptake inhibitors, sulfonamides, methotrexate, triamterene, sulfasalazine, estrogen, and phenytoin; and non-compliance with treatment when $>15 \%$ to $20 \%$ deviation of consumption existed.

\section{Procedure}

The Greene Scale was first completed by the subjects referred to the Helal-e-Ahmar clinic. If the scores were between 15 and 42, written consent for participation in study was obtained after explaining the re- 
search objectives. Subject enrollment was based on the inclusion and exclusion criteria. To hide the allocation and maintain double-sided blinding throughout the study, Vitex agnus-castus extracts and placebo were put in identical envelopes and opaque packages with serial continuous numbers. In each envelope were 30-mg Vitex and placebo medications for 8 weeks' consumption. To comply with the doubleblinding, neither the clinician nor the participants knew about who belonged to the Vitex or placebo group. During the first visit and after basic evaluations, each participant received a numbered envelope based on the sequence of entrance of the participant, and the process continued for 8 weeks. A phone call was made 4 weeks after the start to remind the participants to regularly use the medication. In this study, Vitex agnus-castus extract was administered in capsules. The Vitex extract and placebo were produced by Poursina Pharmacy Company in Tehran. The capsules of Vitex agnus-castus extract (with effective components of trans-anethole, fenchone, methyl chavicol, and estragole standardized based on 21-27 anetholes in each capsule) and placebo (lactose powder, $5 \%$ gelatine solution, and avicel) were identical in shape, size, color, and smell. The regimen consisted of twice-daily ingestion (morning and evening) of Vitex and placebo capsules, and the code of each medication used was recorded in the participant's questionnaire sheet. Undesirable side effects were recorded during each visit by a skilled physician based on a PRD herbal book. In case of any side effects, administration of the medication was ceased, and the subject was excluded from the study.

\section{Measurement Instruments}

The Greene Questionnaire: This scale was developed by Greene in 1975 in Scotland, and its validity and reliability have been confirmed. ${ }^{21)}$ This questionnaire includes 21 items related to menopausal complications in five aspects: anxiety (items 1-6), depression (items 7-11), somatic (items 12-18), vasomotor dysfunction (items 19-20), and sexual dysfunction (items 21). Each symptom was scored by the subject. Scores are assigned as follows: not at all, 0; a little, 1; quite a bit, 2; and extremely, 3; this results in a total score ranging from 0 to 63 . One question was on the number of hot flashes. ${ }^{22)}$

\section{Ethical Considerations}

This study was approved by the Medical Research and Ethics Committee of Kermanshah University of Medical Sciences, Kermanshah, Iran on October 19, 2016 (registration no., Kums.REC.1395.44). Each participant was verbally provided with information regarding the study and the contents of the information sheet. All participants signed a consent form in which the study procedures were explained.

\section{Statistical Analyses}

The Kolmogorov-Smirnov test was used to analyze quantitative data. After confirming data that were normally distributed, analytical statistical methods of the independent and paired T-tests were used to compare the means of quantitative variables between the two groups and within each group. To find an association between the qualitative variables, the chi-square test was performed. All analyses were performed using the IBM SPSS ver. 20.0 statistical software (IBM Corp.,

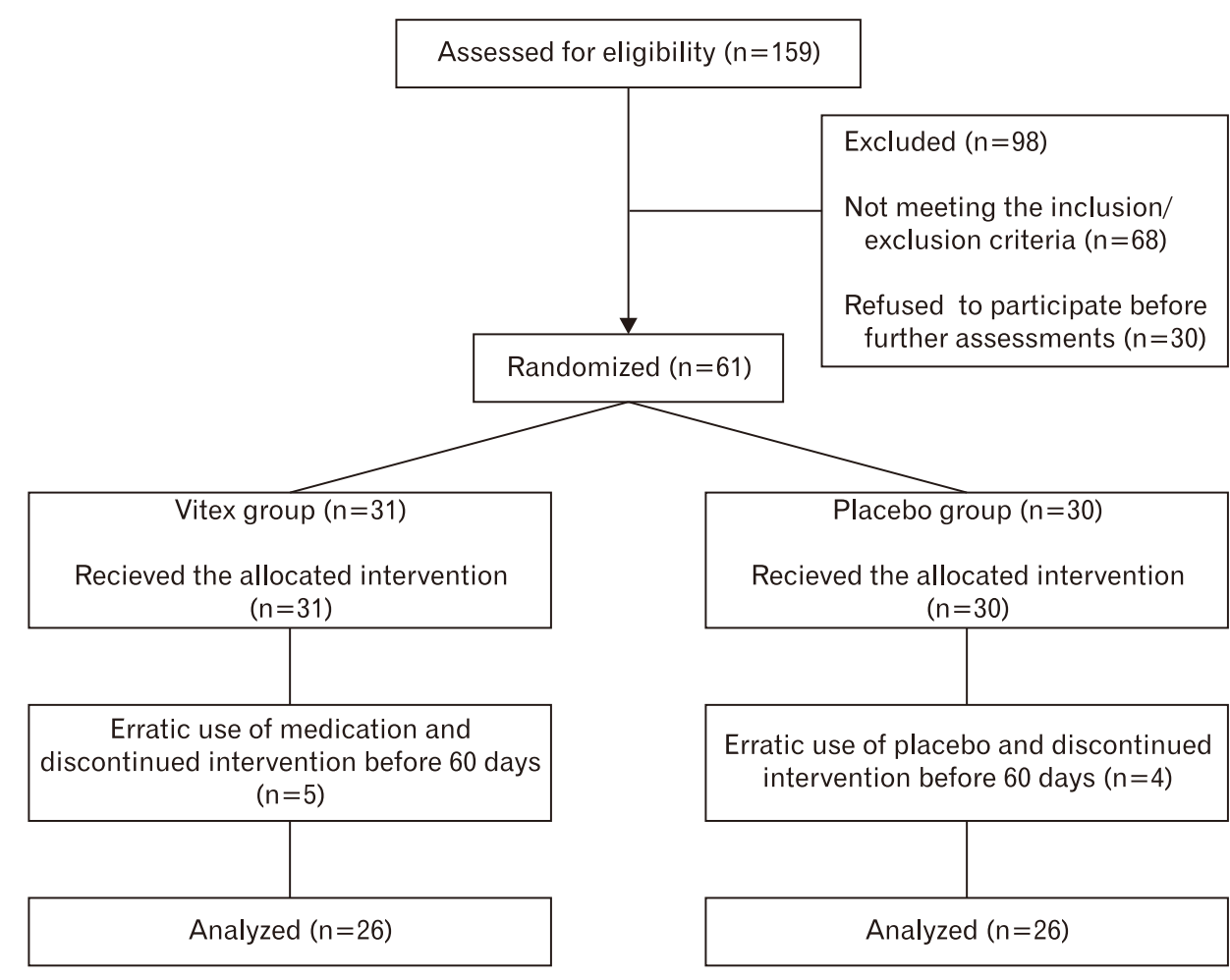

Figure 1. CONSORT diagram showing the flow of participants through each stage. 
Armonk, NY, USA) at a level of error of $5 \%$.

\section{RESULTS}

In this randomized double-blind clinical trial, 159 eligible subjects were evaluated, of whom 98 were excluded ( 68 based on the inclusion and exclusion criteria, and 30 owing to reluctance). Sixty-one subjects were randomly assigned to the two groups, the Vitex group $(n=31)$ and placebo group ( $\mathrm{n}=30)$, and were followed up for 8 weeks.

During the study, five subjects in the Vitex group (due to side effects) and four in the treatment group (due to side effects and unwillingness to continue to use) dropped out. A total of 26 subjects each were randomly allocated to the Vitex and placebo groups and analyzed (CONSORT flow diagram) (Figure 1).

The age range of the participants was $48-58$ years. The mean ages in the Vitex ( 52.85 years) and placebo groups ( 52.16 years) were not statistically significant $(\mathrm{P}=0.38)$. In the Vitex group, 23 (88.05) were unemployed and 3 (11.5) were employed. In the placebo group, 24 (92.04) were housewives and 2 (7.06) were employed. No statistically significant relationship was found between employment status and the study groups $(\mathrm{P}=0.32)$. In terms of marital status, in the Vitex group, 2 subjects (7.7) were single and 24 (92.03) were married. In addition, in the placebo group, 2 (7.7) were single and 24 (92.03) were married. No significant difference in the distribution of marital status was observed between the two groups ( $\mathrm{P}=0.52)$. In addition, no significant relationship was found between income level and mean body mass index in the study groups $(\mathrm{P}=0.16, \mathrm{P}=0.52$, respectively). A number of years divorced of 10-18 years was reported in the two groups, with a mean of 13.76 in the Vitex group and 13.50 in the placebo group. No significant difference was found between the two groups $(\mathrm{P}=0.58)$. The pregnancy rate was also reported, with a mean of 3.77 in the Vitex group and 3.74 in the placebo group $(\mathrm{P}=0.95)$. The mean difference was not significant between the two groups $(\mathrm{P}=0.95)$. The menopausal age was reported in the two groups to be between the ages of 46 to 57 years, the mean of which was not significantly different between the Vitex group (50.94) and placebo group (50.84; $\mathrm{P}=0.89$ ). The years elapsed since menopause, with a variation range of 1-3 years, was also higher in the Vitex group than in the placebo group (1.32), with a statistically significant difference $(\mathrm{P}<0.05)$. The number of hot flashes per day with a variation range of 3 to 20 times was reported in the Vitex and placebo groups, with means of 8.58 and 9.24, respectively, which were not statistically significantly different between the two groups $(\mathrm{P}=0.74)$. As a result of Vitex and placebo use, 3 subjects (11.53) in the Vitex group experienced nausea and 2 (7.69) experienced itching. In the placebo group, only 1 subject (3.84) had nausea, with no significant difference in the incidence of side effects between the two groups $(\mathrm{P}=0.07)$ (Table 1).

The results of the comparison of the mean scores related to the menopausal symptoms at the beginning of the study between the Vitex and placebo groups by the independent-samples T-test (differences with significance level of $<5 \%$ were significant) (Table 2), including anxiety, depression, somatic complications, vasomotor dysfunction, sexual dysfunction, and total dysfunction, showed that in the Vitex group, the mean scores were higher. However, this comparison between complications was not statistically meaningful at this point (i.e., before the intervention; $\mathrm{P}>0.05$ ).

After 8 weeks of follow-up and at the end of study, the results of the comparison between the Vitex and placebo groups regarding the mean scores of menopausal complications showed that the means were lower in the Vitex group for all complications.

The comparison of these results showed that at a margin of error of $5 \%$, the mean scores for anxiety and for vasomotor and total dysfunctions were significantly different between the Vitex and placebo groups $(\mathrm{P}<0.0001)$, leaving decreases in the mean sores for anxiety and vasomotor and total dysfunction to 3.2 and 3 times lower in the treatment

Table 1. Demographic of the treatment and placebo groups

\begin{tabular}{|c|c|c|c|c|}
\hline Variable & Levels & Vitex & Placebo & P-value \\
\hline Age (y) & $48-58$ & $52.85 \pm 2.70$ & $52.16 \pm 2.80$ & 0.38 \\
\hline \multirow[t]{2}{*}{ Occupation } & Housekeeper & 23 (88.05) & $24(92.04)$ & 0.32 \\
\hline & Employed & $3(11.5)$ & $2(7.06)$ & \\
\hline \multirow[t]{2}{*}{ Marital status } & Single & $2(7.7)$ & $2(7.7)$ & 0.52 \\
\hline & Married & 24 (92.03) & 24 (92.03) & \\
\hline \multirow[t]{3}{*}{ Income } & Less than US\$ 300 monthly income & $12(46.2)$ & $18(69.3)$ & 0.16 \\
\hline & US\$ 300 to 900 monthly income & $12(46.2)$ & $8(30.7)$ & \\
\hline & More than US\$900 monthly income & $2(7.6)$ & 0 & \\
\hline Body mass index $\left(\mathrm{kg} / \mathrm{m}^{2}\right)$ & $1.75-3$ & $2.30 \pm 0.35$ & $2.40 \pm 0.25$ & 0.17 \\
\hline Years after menopause & $1-3$ & $2.02 \pm 0.78$ & $1.32 \pm 0.53$ & $0.001^{\star *}$ \\
\hline No. of hot flashes & $3-20$ & $8.58 \pm 5.40$ & $9.24 \pm 8.40$ & 0.74 \\
\hline Menopausal age (y) & $46-57$ & $50.94 \pm 2.53$ & $50.84 \pm 2.54$ & 0.89 \\
\hline Menarche age (y) & $10-18$ & $13.76 \pm 1.58$ & $13.50 \pm 1.69$ & 0.58 \\
\hline No. of deliveries & $1-9$ & $3.77 \pm 1.51$ & $3.74 \pm 1.76$ & 0.95 \\
\hline \multirow[t]{2}{*}{ Adverse effects } & Nausea & $3(11.53)$ & $1(3.84)$ & 0.07 \\
\hline & Itching & $2(7.69)$ & 0 & \\
\hline
\end{tabular}

Values are presented as mean \pm standard deviation or number (\%).

${ }^{\star *} \mathrm{P}<0.01$. 
Table 2. Comparison of the means of the menopausal symptoms based on Green's questionnaire before and after the intervention between the Vitex extract and placebo groups

\begin{tabular}{|c|c|c|c|c|c|c|}
\hline \multirow{2}{*}{ Variable } & \multicolumn{2}{|c|}{ Before intervention } & \multirow{2}{*}{ Significance } & \multicolumn{2}{|c|}{ After the intervention } & \multirow{2}{*}{ P-value } \\
\hline & Vitex & Placebo & & Vitex & Placebo & \\
\hline \multicolumn{7}{|l|}{ Menopausal symptoms } \\
\hline Anxiety & $9.65 \pm 3.12$ & $8.36 \pm 2.43$ & 0.11 & $2.35 \pm 1.52$ & $6.28 \pm 2.88$ & $<0.001^{* \star}$ \\
\hline Depression & $8.04 \pm 2.47$ & $7.52 \pm 2.29$ & 0.44 & $4.61 \pm 2.87$ & $5.04 \pm 2.80$ & 0.60 \\
\hline Somatic complications & $7.08 \pm 3.28$ & $5.60 \pm 3.25$ & 0.11 & $3.50 \pm 2.12$ & $4.24 \pm 3.09$ & 0.32 \\
\hline Vasomotor & $3.73 \pm 1.71$ & $3.76 \pm 1.64$ & 0.95 & $0.46 \pm 0.90$ & $2.72 \pm 1.54$ & $<0.001^{\text {** }}$ \\
\hline Sexual dysfunction & $1.65 \pm 0.84$ & $1.56 \pm 1.23$ & 0.75 & $1.07 \pm 0.69$ & $1.44 \pm 1.08$ & 0.16 \\
\hline Total & $29.54 \pm 7.29$ & $26.88 \pm 4.85$ & 0.13 & $12.15 \pm 4.31$ & $20.12 \pm 6.64$ & $<0.001^{* *}$ \\
\hline
\end{tabular}

Values are presented as mean \pm standard deviation.

${ }^{* *} \mathrm{P}<0.01$.

group, respectively. On the other hand, at the end of study, the mean scores for depression, somatic complications, and sexual dysfunction variables had no significant statistical differences between the Vitex and placebo groups (Table 2).

\section{DISCUSSION}

This research was conducted to determine the effect of the Vitex plant extract on menopausal symptoms in comparison with that of placebo. Results showed that in the Vitex group, the total scores for menopause, anxiety, and vasomotor dysfunction were meaningfully diminished after the intervention. The notable point was that along with the Vitex group, the placebo group showed decreased mean scores for menopausal symptoms, which could be attributed to the suggestibility effect in this group, although the recovery process of the menopausal symptoms in the Vitex group was significantly more prominent that that in the placebo group.

The results of this study are congruent with those of the study by van Die et al. ${ }^{16)}$ in 2009 that reported the effectiveness of Vitex agnus-castus extracts in the treatment of menopause-related complaints. They are also in agreement with the results of the study by Abbaspoor et al. ${ }^{20}$ who conducted a double-blind clinical trial in 2011 to assess the effects of Vitex on primary menopausal symptoms and revealed that Vitex was effective in reducing primary vasomotor symptoms in women. Berger et al. ${ }^{23)}$ in 2000 also showed that Vitex was effective for menopausal symptoms. In explaining this finding, the mechanism of the main effect of the plants in the treatment of some female diseases seems to be their effects on body hormones. Studies have demonstrated that some important plant components, including flavonoids, directly affect the pituitary gland, especially the part of the gland that secretes luteinizing hormone, which increases the progesterone level and modify disorders caused by decreases in hormone levels. Previous studies reported that Vitex can decrease prolactin levels in rats. This study also mentioned that Vitex can inhibit prolactin release by binding to dopamine receptors. ${ }^{24,25)}$

Vitex contains phytoestrogens, which are weak estrogen agonists; therefore, it can be conceptualized that in low-estrogen environments, similar to that in as in menopausal women, this plant can exert more radical estrogenic effects. ${ }^{26)}$

Vasomotor dysfunction is the most problematic symptom in menopause. The prevalence of vasomotor dysfunction in European, Malaysian, Chinese, and Singaporean menopausal women is estimated to be $70 \%-80 \%, 18 \%$, and $14 \%$, respectively, which is attributed to the differences in dietary phytoestrogen levels in these areas. ${ }^{27)}$ In a randomized controlled assessment by Knight and Eden ${ }^{28)}$ in 1995, decreasing trends in the prevalence rates of headache, sleep problems, depression, and irritability were observed among users of phytoestrogencontaining diets, denoting the fact that phytoestrogens can play a role in reducing menopausal symptoms, similar to Vitex. No significant differences in the mean scores of sexual dysfunction, physical complications, and depression were found between the Vitex and placebo groups. In the explanation of this finding, it can be told that sexual contact in menopause, in addition to physiological factors, are affected by individual, social, and psychological factors. Some women in their menopausal phase are less concerned about their sexual relationships and have less sexual activity, ${ }^{29)}$ which can affect the outcome of the treatment.

In addition, in the explanation for the same score for physical complications and depression in the Vitex and placebo groups, some stressors such as family problems, negative attitudes to physical changes, and factors associated with menopause may be confounding. We can conclude that in the treatment of menopausal symptoms, psychological and social factors should be considered in therapeutic approaches. Unfortunately, in the present study, it was emphasized only in the biological dimensions.

Although this study showed that Vitex can be effective in alleviating menopausal symptoms, further research is essential to clarify the degree of effectiveness and the mechanisms of action of Vitex.

Some limitations of this study were the small number of subjects, the absence of follow-up findings, and use of a questionnaire to record menopausal symptoms that can potentially give rise to respondent bias. It is advisable to use larger sample sizes and follow the results of interventions.

In conclusion, Vitex plant extract can be considered as an inexpensive, effective, and safe treatment with no side effects that can be applied as a non-hormonal remedy option along with other therapy 
methods available for menopausal complications.

\section{CONFLICT OF INTEREST}

No potential conflict of interest relevant to this article was reported.

\section{ACKNOWLEDGMENTS}

The authors thank the Substance Abuse Prevention Research Center and the Clinical Research Development Unit of Emam Reza Hospital, University of Medical Sciences, Kermanshah, Iran, for their support, cooperation, and assistance throughout the study period (grant no., 96062).

\section{ORCID}

Rozita Naseri: https://orcid.org/0000-0002-5724-3455

Vahid Farnia: https://orcid.org/0000-0003-0666-453X

Katayoun Yazdchi: https://orcid.org/0000-0002-1742-7313

Mostafa Alikhani: https://orcid.org/0000-0001-6848-3027

Behrad Basanj: https://orcid.org/0000-0002-1109-4095

Safora Salemi: https://orcid.org/0000-0002-4988-2521

\section{REFERENCES}

1. Norozi A, Kasiri N, Aslami A. Attitudes and perceptions of women 45 years of menopause. J Health Syst Res 2010;7:14.

2. Bachmann GA. Menopausal vasomotor symptoms: a review of causes, effects and evidence-based treatment options. J Reprod Med 2005;50:155-65.

3. Tanira S, Wazed F, Sultana A, Amin R, Sultana K, Ahmad S. Knowledge, attitude and experience of menopause an urban based study in Bangladesh. J Dhaka Med Coll 2009;18:33-6.

4. Dennerstein L, Lehert P, Guthrie JR, Burger HG. Modeling women's health during the menopausal transition: a longitudinal analysis. Menopause 2007;14:53-62.

5. Dennerstein L. Well-being, symptoms and the menopausal transition. Maturitas 1996;23:147-57.

6. Abdolahi F, Shabankhani B, Zarkhami M. Women of menopausal age in Mazandaran province in 2001. J Mazandaran Univ Med Sci 2004; 14:61-8.

7. Pan HA, Wu MH, Hsu CC, Yao BL, Huang KE. The perception of menopause among women in Taiwan. Maturitas 2002;41:269-74.

8. Hasanzadeh GH, Zareh S, Vaziri S, Sarvari Rad B, Dargi L. Women's knowledge about menopause in Qazvin. J Qazvin Univ Med Sci 2003; 2:21-5.

9. Schott-Baer D, Kotal B. Frequency and effectiveness of self-care actions and menopause symptoms of middle-aged working women. Medsurg Nurs 2000;9:302-7.

10. Van der Mooren MJ, Kenemans P. Postmenopausal hormone therapy: impact on menopause-related symptoms, chronic disease and quality of life. Drugs 2004;64:821-36.

11. Jing Z, Yang X, Ismail KM, Chen X, Wu T. Chinese herbal medicine for premenstrual syndrome. Cochrane Database Syst Rev 2009;(1): CD006414.

12. Amirghofran Z, Bahmani M, Azadmehr A, Javidnia K, Miri R. Immunomodulatory activities of various medicinal plant extracts: effects on human lymphocytes apoptosis. Immunol Invest 2009;38:181-92.

13. Salehian T, Safdari F, Piry A, Atarody Z. Herbal remedy to relieve of dysmenorrhea by students of Iranshahr Universities in 2010. J Herb Drugs 2011;1:57-63.

14. Ramezani M, Amin G, Jalili E. Antinociceptive and anti-inflammatory effects of hydroalcoholic extract of Vitex agnus castus fruit in mice. J Shahrekord Univ Med Sci 2010;11:46-51.

15. Nasri S, Ebrahimi S. Medical effect of Vitex agnus-castus. J Babol Univ Med Sci 2006; 7:49-53.

16. Van Die MD, Burger HG, Teede HJ, Bone KM. Vitex agnus-castus (Chaste-Tree/Berry) in the treatment of menopause-related complaints. J Altern Complement Med 2009;15:853-62.

17. Najar S, Yaralizadeh M, Abedi P, Namjooyan F, Malehi O. Effect of Fennel vaginal cream on dysparonia and sexual satisfaction among postmenopausal women: a double-blind randomized controlled trial. Iran J Obstet Gynecol Infertil 2015;18:8-16.

18. Abdali K, Dowran P, Emamghoreishi M, Kasraian M, Tabatabaei H. Comparison of the effect of Foeniculum vulgare and St John's wort (Hypericum perforatum) on the climacteric symptoms and sexual activity in menopausal woman. Int J Adv Biotechnol Res 2016;7:148-54.

19. Yaralizadeh M, Abedi P, Najar S, Namjoyan F, Saki A. Effect of Foeniculum vulgare (fennel) vaginal cream on vaginal atrophy in postmenopausal women: a double-blind randomized placebo-controlled trial. Maturitas 2016;84:75-80.

20. Abbaspoor Z, Hajikhani NA, Afshari P. Effect of Vitex agnus-castus on menopausal early symptoms in postmenopausal women: a randomized, double blind, placebo-controlled study. Br J Med Med Res 2011; 1:132-40.

21. Nahidi F, Taherpour M, Mojab F, Alavi Majd H. Effects of Anise abstract on menopausal hot flashes. Pajoohandeh J 2008;13:167-73.

22. Abdali K, Dowran P, Emamghoreishi M, Kasraian M, Tabatabaei H. Comparison of the effect of Foeniculum vulgare and St John's wort (Hypericum perforatum) on the climacteric symptoms and sexual activity in menopausal woman. Int J Adv Biotechnol Res 2016;7:148-54.

23. Berger D, Schaffner W, Schrader E, Meier B, Brattstrom A. Efficacy of Vitex agnus castus L. extract Ze 440 in patients with pre-menstrual syndrome (PMS). Arch Gynecol Obstet 2000;264:150-3.

24. Delaram M, Sadeghiyan Z. The effect of echinophora-platyloba extract on primary of dysmenorrhea. Arak Med Univ J 2010;13:61-7.

25. Namavar Jahromi B, Tartifizadeh A, Khabnadideh S. Comparison of fennel and mefenamic acid for the treatment of primary dysmenorrhea. Int J Gynaecol Obstet 2003;80:153-7.

26. Davis SR, Murkies AL, Wilcox G. Phytoestrogens in clinical practice. Integr Med 1998;1:27-34.

27. Warren MP. The effects of phytoestrogen supplementation in postmenopausal women. J Soc Gynecol Investig 2002;9:184-5.

28. Knight DC, Eden JA. Phytoestrogens: a short review. Maturitas 1995; 22:167-75.

29. Moghassemi S, Ziaei S, Haidari Z. Female sexual dysfunction in Iranian postmenopausal women: prevalence and correlation with hormonal profile. J Sex Med 2011;8:3154-9. 